\title{
Temporal-Based Risk Forecasting Approach for Key Areas on Surveillance Sensor Networks of High- Speed Railway Transport Hub
}

\author{
Xie Zhengyu \\ School of Traffic and Transportation \\ Beijing Jiaotong University \\ Beijing, China \\ xiezhengyu@bjtu.edu.cn
}

\author{
Qin Yong \\ State Key Laboratory of Rail Traffic Control and Safety \\ Beijing Jiaotong University \\ Beijing, China \\ qingyong2146@126.com
}

\author{
Wang Li \\ School of Traffic and Transportation \\ Beijing Jiaotong University \\ Beijing, China \\ liwang@bjtu.edu.cn
}

\begin{abstract}
Key areas risk forecasting plays an important roles in safety management in high-speed railway transport hub. In this paper, a temporal-based risk forecasting approach was considered for key areas on surveillance sensor networks. Computational experiments on a specific key area in high-speed railway transport hub were conducted to illustrate the proposed approach. The results showed the temporal-based forecasting approach is effective and efficient for key areas risk forecasting in high-speed railway transport hub.
\end{abstract}

Keywords- Surveillance Sensor Networks; Temporal-Based, Forecasting; High-speed railway transport hub

\section{INTRODUCTION}

In the last ten years, China high-speed railway had a rapid development. At the end of 2015, a large high-speed railway network has been built with lines in operation amounting to $19,000 \mathrm{~km}$. As an important transferring node, high-speed railway transport hub combines with urban rail transit, taxi, private vehicle, airplane, bus, etc. and has huge passenger distributed every day. Massive passenger flow in high-speed railway transport hub may cause crowd massing situation which bring several potential risks to the safety management of high-speed railway transport hubs.

At present, video surveillances are widely applied in highspeed railway transport hubs for monitoring and detecting passenger status of key areas. Based on the surveillance sensors networks, many approaches for in high-speed railway transport hub safety were proposed in literature.

Several passenger image processing and intelligent detecting algorithms were developed for rapid and accurate detection of passenger flow status [1-4]. To improve detection accuracy in terms of feature extraction, a novel feature of gradient self-similarity (GSS) was present, which was computed from HOG, and was applied to capturing the patterns of pairwise similarities of local gradient patches [1]. In order to accurately and promptly detect potential safety hazard, a modified background model based on Dempster-Shafer theory, and a passenger flow status recognition algorithm based on features of image connected domain were proposed to improve the accuracy and real-time performance of passenger flow detection [2]. For the challenging of abrupt illumination, occlusions, out of field of view, and cluttered backgrounds, a novel tracking framework was developed, which consists of two steps: image shadow removal and tracking by association [3]. A sparse representation based approach is proposed for pedestrian detection from thermal images. The approach first adopted the histogram of sparse code to represent image features and then detect pedestrian with the extracted features in an unimodal and a multimodal framework respectively [4].

Some studies focused on causing and handling of the potential risks [5-7]. The selfish and selfless behaviors were considered as two main factors in evacuation, and selfishnessand selflessness-based model of pedestrian room evacuation was proposed [5]. A multi-grid model was proposed to simulate evacuation with guiders, and the effects of guider type, guider number, guider distribution and guidance strategy on evacuation were discussed [6]. A pedestrian evacuation simulation model based on the extended cellular automata was proposed with the consideration of heterogeneous behavioral tendencies in humans, and the model was applied to optimize buildings to reduce evacuation times [7].

According to literature review above, current studies mainly focused on risks detection and handling. Specific literature on risk forecasting is scarce. In order to accurately detect potential safety hazard hidden in passenger flow, a 
hybrid forecasting approach was proposed to forecast the passenger flow status [8]. This approach was only for passenger amount forecasting, and did not consider area risk. Based on the surveillance sensor networks, a passenger flow risk forecasting algorithm based on spatial correlation was developed [9]. The approach was based on the spatial correlation between key area and related areas, and did not consider influence from passenger flow risk temporal change in key area. So in this paper, based on surveillance sensor networks, we forecast passenger flow risk in key area by using itself temporal risk change, and a temporal-based risk forecasting algorithm is developed.

The rest of this paper is organized as follows: The key area risk value calculation is introduced in section 2 and section 3 proposes a temporal-based passenger flow risk forecasting algorithm. Numerical experiments are conducted in section 4 and finally Section 5 covers the conclusion.

\section{KEY AREA RISK VALUE CALCULATION IN IN HIGH-SPEED RAILWAY TRANSPORT HUB}

Currently, most of high-speed railway transport hubs calculate key area risk by using the passenger flow amount in key area. In the calculation process, three type passenger flow status parameters are adopted. First is current passenger flow amount of key area $A_{1}$, which can be real-timely obtained by surveillance sensors in key area. Second is average passenger flow amount of key area in statistic period $A_{2}$ and third is max passenger flow amount of key area in statistic period $A_{3}$. The last two parameters can be obtained from historical passenger flow amount of key area in statistic period. According to the different relationship among $A_{1}, A_{2}, A_{3}$, the key area risk value $r(x)$ can be calculated as follows [9]:

(i) While $A_{1} \leq A_{2}, r(x)$ can be calculated by Eq.(1).

$$
r(x)=3-10\left(\frac{A_{2}-A_{1}}{A_{2}}\right)
$$

(ii) While $A_{2}<A_{1} \leq A_{3}, r(x)$ can be calculated by Eq.(2).

$$
r(x)=\frac{4 A_{1}+3 A_{3}-7 A_{2}}{A_{3}-A_{2}}
$$

(iii) While $A_{1}>A_{3}, r(x)$ can be calculated by Eq.(3).

$$
r(x)=7+10\left(\frac{A_{1}-A_{3}}{A_{3}}\right)
$$

\section{TEMPORAL-BASED PASSENGER FLOW RISK FORECASTING ALGORITHM}

In this section, a temporal forecasting algorithm is proposed to forecast key area risk based on radial basis function neural network in high-speed railway transport hubs.

\section{A. Radial basis function neural network (RBF NN)}

RBF NN is a typical feed-forward neural network, which has many merits, such as nonlinear mapping characteristics, self-organized study ability, training fast, and the capability of converging in global optimization and approaching the function in the best way [8]. Simply for its great advantages, RBFNN has been applied in many fields. So in this paper, we propose a temporal forecasting approach based on RBF $\mathrm{NN}$ to forecast key area risk in high-speed railway transport hubs.

\section{B. RBF NN design for key area risk forecasting}

There are three different layers in the structure of RBF NN, which are input layer, hidden layer and output layer. The structure of RBF NN for temporal-based key area risk forecasting is shown in Fig.1.



Figure 1. RBF NN structure for temporal-based key area risk forecasting

Main step for RBF NN design is shown as follows:

1) Sample data processing: in order to ensure the input value in the range of 0 and 1 , a normalization is need to process the input data. For the time series $X$, we use Eq.(4) for normalization processing.

$$
x_{i}^{\prime}=k\left(x_{i}-\min X\right) /(\max X-\min X)
$$

Where $\min X$ is the minimum value in time series $X$, $\max X$ is the maximum value in time series $X$ and $k$ is samples normalized scale.

2) Input vector determine: in statistic period, the key area risk values can be regarded as a time series $x=\left\{x_{i} \mid x_{i} \in R, i=1,2, \ldots, L\right\}$, and we can use the risk values before time epoch $N$ to forecast the risk value at time epoch $M$. Table I is a mapping structure of time series.

TABLE I. MAPPING STRUCTURE OF TIME SERIES

\begin{tabular}{|c|c|}
\hline N input value & M output value \\
\hline$x_{1}, x_{2}, . ., x_{N}$ & $x_{N+1}, x_{N+2}, . ., x_{N+M}$ \\
\hline$x_{2}, x_{3}, . ., x_{N+1}$ & $x_{N+2}, x_{N+3}, . ., x_{N+M+1}$ \\
\hline
\end{tabular}




\begin{tabular}{|c|c|}
\hline N input value & Moutput value \\
\hline$\ldots \ldots$ & $\ldots \ldots$ \\
\hline$x_{K}, x_{K+1}, . ., x_{N+K-1}$ & $x_{N+K}, x_{N+K+1}, \ldots, x_{N+M+K+1}$ \\
\hline
\end{tabular}

3) Network parameter: according to a large number of experiments, we finally adopt gauss basis function as the function of hidden layer, k-means clustering algorithm as the center traning method.

\section{NUMERICAL EXPERIMENTS}

In this section, numerical experiments are conducted to verify the proposed algorithm. We take a specific key area in high-speed railway transport hub as an example, select time period from 12:00 to $18: 00$ in a festival day to conduct experiments. We set 10 minutes as a forecasting period, and make a comparison between forecasting risk value and actual risk value. The comparison results are shown in Table II and Fig.2, and the GAPs between forecasting risk value and actual risk value are shown in Fig.3.

TABLE II. COMPARISON BETWEEN FORECASTING VALUE AND ACTUAL VALUE

\begin{tabular}{|c|c|c|c|c|}
\hline Hour & Period & $\begin{array}{l}\text { Forecasting } \\
\text { risk value }\end{array}$ & $\begin{array}{l}\text { Actual risk } \\
\quad \text { value }\end{array}$ & GAP \\
\hline \multirow{6}{*}{ 12:00 } & 1 & 7 & 6.8 & $2.94 \%$ \\
\hline & 2 & 6.9 & 7 & $-1.43 \%$ \\
\hline & 3 & 7.4 & 7.2 & $2.78 \%$ \\
\hline & 4 & 6.9 & 7.2 & $-4.17 \%$ \\
\hline & 5 & 7.3 & 7.6 & $-3.95 \%$ \\
\hline & 6 & 7.2 & 7.4 & $-2.70 \%$ \\
\hline \multirow{6}{*}{$13: 00$} & 7 & 7.2 & 6.8 & $5.88 \%$ \\
\hline & 8 & 7.1 & 6.8 & $4.41 \%$ \\
\hline & 9 & 6.7 & 6.5 & $3.08 \%$ \\
\hline & 10 & 6.3 & 6.6 & $-4.55 \%$ \\
\hline & 11 & 6.7 & 6.8 & $-1.47 \%$ \\
\hline & 12 & 7 & 7 & $0.00 \%$ \\
\hline \multirow{6}{*}{ 14:00 } & 13 & 7.1 & 7.3 & $-2.74 \%$ \\
\hline & 14 & 7.5 & 7.6 & $-1.32 \%$ \\
\hline & 15 & 7.9 & 6 & $31.67 \%$ \\
\hline & 16 & 8 & 6.4 & $25.00 \%$ \\
\hline & 17 & 7.8 & 6.3 & $23.81 \%$ \\
\hline & 18 & 7.9 & 6.7 & $17.91 \%$ \\
\hline \multirow{6}{*}{$15: 00$} & 19 & 7.7 & 7.2 & $6.94 \%$ \\
\hline & 20 & 7.8 & 7 & $11.43 \%$ \\
\hline & 21 & 6.9 & 6.6 & $4.55 \%$ \\
\hline & 22 & 6.8 & 6.7 & $1.49 \%$ \\
\hline & 23 & 7 & 6.9 & $1.45 \%$ \\
\hline & 24 & 7.2 & 7 & $2.86 \%$ \\
\hline \multirow{6}{*}{ 16:00 } & 25 & 6.9 & 7.2 & $-4.17 \%$ \\
\hline & 26 & 7.1 & 7.4 & $-4.05 \%$ \\
\hline & 27 & 7.4 & 7.4 & $0.00 \%$ \\
\hline & 28 & 7.6 & 7.8 & $-2.56 \%$ \\
\hline & 29 & 7.8 & 7.6 & $2.63 \%$ \\
\hline & 30 & 7.3 & 6.9 & $5.80 \%$ \\
\hline \multirow{6}{*}{ 17:00 } & 31 & 7.5 & 6.9 & $8.70 \%$ \\
\hline & 32 & 7 & 6.6 & $6.06 \%$ \\
\hline & 33 & 6.8 & 6.7 & $1.49 \%$ \\
\hline & 34 & 6.9 & 6.8 & $1.47 \%$ \\
\hline & 35 & 7.2 & 7 & $2.86 \%$ \\
\hline & 36 & 7 & 7.3 & $-4.11 \%$ \\
\hline
\end{tabular}

Note: $\mathrm{GAP}=($ forecasting risk value - actual risk value $)$ / actual risk value* $100 \%$

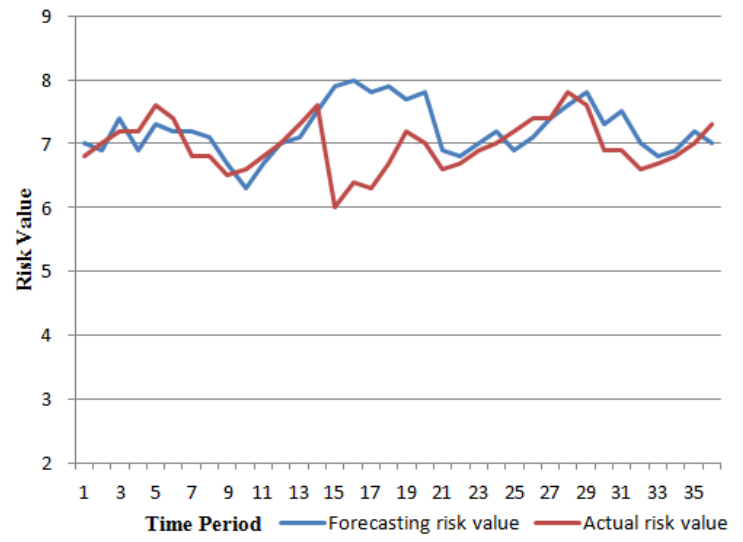

Figure 2. Comparison between forecasting risk value and actual risk value

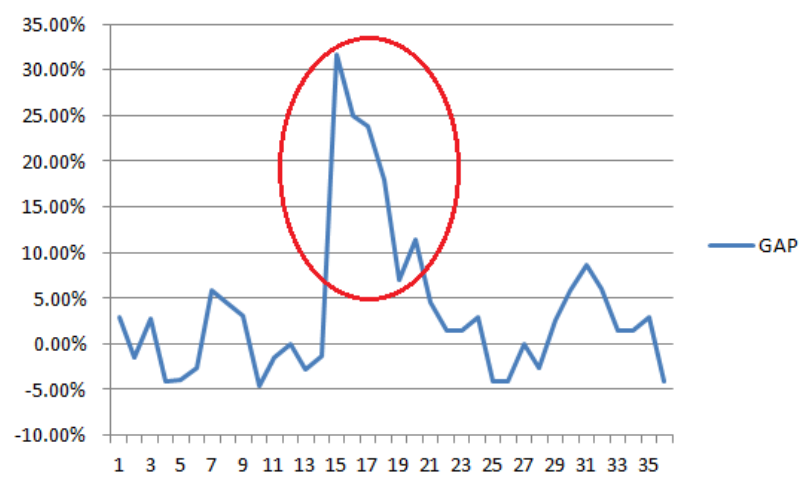

Figure 3. GAP between forecasting risk value and actual risk value

As observed in Table II and Fig.2, the forecasting risk values obtained by our approach are close to actual risk value, except some specific time period. The average gap between forecasting risk value and actual risk value is $3.83 \%$. The variation trend of passenger flow risk is mainly well forecasted by our approach. In the time period 15 to 18 , the GAPs forecasting risk value and actual risk value are prominent higher than average GAP (red ellipse area in Fig.3). The forecasting errors result from the passenger flow congestion in related areas of the key area. Our approach is insensitive for congestion situation. Generally, our forecasting approach has a good performance for mass passenger flow in festival days, and can meet the demands of safety management in high-speed railway transport hub.

\section{CONCLUSION}

In this paper, we considered key area risk forecasting problem in high-speed railway transport hub. Based on the surveillance sensor network of hub, a temporal-based passenger flow risk forecasting algorithm was developed. Numerical experiments on a key area in high-speed railway transport hub showed that the proposed forecasting approach is 
effective to forecast key area risk in hub. In future, for our forecasting approach, solving the insensitiveness for passenger flow congestion is an important task for further research.

\section{ACKNOWLEDGMENT}

This research was supported by the Fundamental Research Funds for the Central Universities (Grant no. 2015JBM044), the Talented Faculty Funds of Beijing Jiaotong University (Grant no. 2014RC005) and State Key Laboratory Program (Grant no. RCS 2016ZT016)

\section{REFERENCES}

[1] Xie Z Y, Jia L M, Qin Y, et al, "Passenger Flow Detection of Video Surveillance: A Case Study of High-Speed Railway Transport Hub in China," Elektronika ir Elektrotechnika, vol.21, pp. 48-53. 2015.

[2] Wu S, Laganière R, Payeur $\mathrm{P}$, "Improving pedestrian detection with selective gradient self-similarity feature," Pattern Recognition, vol.48, pp. 2364-2376, 2015.
[3] Shen X, Sui X, Pan K, et al, "Adaptive pedestrian tracking via patchbased features and spatial-temporal similarity measurement," Pattern Recognition, vol.53, pp. 163-173, 2015.

[4] Qi B, John V, Liu Z, et al, "Pedestrian detection from thermal images: A sparse representation based approach," Infrared Physics \& Technology, vol.76, pp. 157-167, 2016

[5] Song X, Ma L, Ma Y, et al, "Selfishness-and Selflessness-based models of pedestrian room evacuation," Physica A: Statistical Mechanics and its Applications, vol.447, pp. 455-466, 2016.

[6] Cao S, Song W, Lv W, "Modeling pedestrian evacuation with guiders based on a multi-grid model," Physics Letters A, vol. 380, pp. 540-547, 2016.

[7] Li D, Han B, "Behavioral effect on pedestrian evacuation simulation using cellular automata," Safety science, vol.80, pp. 41-55, 2015.

[8] Xie Z Y, Jia L M, Qin Y, et al, "A hybrid temporal-spatio forecasting approach for passenger flow status in chinese high-speed railway transport hub," Discrete Dynamics in Nature and Society, vol.2013, pp. $1-8,2013$.

[9] Xie Z Y, Qin Y, "A Passenger Flow Risk Forecasting Algorithm for High-speed Railway Transport Hub Based on Surveillance Sensor Networks," Journal of Sensors, vol.2016, pp. 1-6, 2016. 